九州大学学術情報リポジトリ

Kyushu University Institutional Repository

\title{
Online algorithms for mining semi-structured data stream
}

Asai, Tatsuya

Department of Informatics, Kyushu University

Arimura, Hiroki

PREST0, JST | Department of Informatics, Kyushu University

Abe, Kenj i

Department of Informatics, Kyushu University

Kawasoe, Shinji

Department of Informatics, Kyushu University

他

http://hdl. handle. net/2324/6228

出版情報: Proceedings of 2002 IEEE International Conference on Data Mining, pp.27-34, 2002. IEEE

バージョン :

権利関係 : ๑ 2002 IEEE 


\title{
Online Algorithms for Mining Semi-structured Data Stream
}

\author{
Tatsuya Asai Hiroki Arimura ${ }^{\dagger}$ Kenji Abe Shinji Kawasoe Setsuo Arikawa \\ Department of Informatics, Kyushu University, Fukuoka 812-8581, JAPAN \\ \{t-asai,arim,k-abe,s-kawa,arikawa\}@i.kyushu-u.ac.jp \\ $\dagger$ PRESTO, JST, JAPAN
}

\begin{abstract}
In this paper, we study an online data mining problem from streams of semi-structured data such as XML data. Modeling semi-structured data and patterns as labeled ordered trees, we present an online algorithm StreamT that receives fragments of an unseen possibly infinite semistructured data in the document order through a data stream, and can return the current set of frequent patterns immediately on request at any time. A crucial part of our algorithm is the incremental maintenance of the occurrences of possibly frequent patterns using a tree sweeping technique. We give modifications of the algorithm to other online mining model. We present theoretical and empirical analyses to evaluate the performance of the algorithm.
\end{abstract}

\section{Introduction}

Recently, a new class of data-intensive applications such as network monitoring, web site management, sensor networks, and e-commerce emerged with the rapid growth of network and web technologies. In these applications, the data are modeled not as static collections but as transient data streams, where the data source is an unbounded stream of individual data items, e.g., transaction records or web page visits, which may arrive continuously in rapid, timevarying way [18].

Particularly in data communication through internet, it is becoming popular to use semi-structured data-based communication technologies [2], e.g., SOAP [19], to send heterogeneous and ill-structured data through networks. Since traditional database technologies are not directly applicable to such data streams, it is important to study efficient information extraction methods for semi-structured data streams.

In this paper, we model such semi-structured data streams by sequences of labeled ordered trees, and study the frequent pattern discovery problem in online setting. We model a semi-structured data stream as an infinite sequence of the nodes generated by the depth-first scanning of a possibly infinite data tree. An online algorithm has to continuously work on the data stream, and has to quickly answer queries on request based on the portion of the data received so far. This formulation captures typical situations for web applications reading a sequence of XML tags or SAX events element by element from a data stream. Since this is a finest-grained online model, the results of this paper can be easily generalized to coarser-grained models where, e.g., XML documents are processed page by page.

We present an online algorithm StreamT for discovering labeled ordered trees with frequency at least a given minimum threshold from an unbounded data stream. A difficulty lies in that we have to continuously work with unbounded data streams using only bounded resources. A key idea is a technique of sweeping a branch, called the sweep branch, over the whole virtual data tree to find all embeddings of candidate patterns intersecting it. Using this sweep branch as a synopsis data structure, we achieve incremental and continuous computation of all occurrences of patterns with bounded working space.

As another idea, we adopt a candidate management policy similar to Hidber [11] for online association mining to limit the number of candidate patterns as small as possible. We also use the enumeration technique for labeled ordered trees that we recently proposed in [4], a generalization of a technique by Bayardo [6]. Combining these ideas, our algorithm StreamT works efficiently in both time and space complexities in online manner. Furthermore, we extend our algorithm to the forgetting model of online data stream mining, where the effect of a past data item decays exponentially fast in its age. We also give theoretical analysis on the accuracy of the discovered patterns as well as an empirical analysis on the scalability of the algorithms.

The rest of this paper is organized as follows. In Section 2, we give basic definitions, and in Section 3, we present our online algorithm. In Section 4, we modify this algorithm in the forgetting model. In Section 5, we report experimental results, and in Section 6, we conclude. For proofs not found here, see [5].

\subsection{Related Works}

Emerging technologies of semi-structured data have attracted wide attention of networks, e-commerce, information retrieval and databases [2, 19]. In contrast, there 
have not been many studies on semi-structured data mining $[1,4,7,9,13,15,16,20,22]$. There are a body of researches on online data processing and mining $[10,14,18]$. Most related work is Hidber [11], who proposed a model of continuous pattern discovery from unbounded data stream, and presented adaptive online algorithm for mining association rules. Parthasarathy et al. [17] and Mannila et al. [14] studied mining of sequential patterns and episode patterns. Yamanishi et al. [21] presented an efficient online-outlier detection system SmartSifter with a forgetting mechanism.

Zaki [22] and Asai et al. [4] independently developed efficient pattern search techniques, called rightmost expansion, for semi-structured data, which is a generalization of the set-enumeration tree technique [6]. Although our algorithm partly uses this technique, its design principle is different from previous semi-structured data mining algorithms $[4,7,9,13,15,16,20,22]$

\section{Preliminaries}

\subsection{Model of Semi-Structured Data}

Semi-structured data are heterogeneous collections of weakly structured data [2], which are typically encoded in a markup language such as XML [19]. We model semistructured data and patterns [2] by labeled ordered trees. For the basic terminologies on trees, we refer to, e.g. [3].

Labeled Ordered Trees. We define labeled ordered trees according to $[4,12]$. Let $\mathcal{L}=\left\{\ell, \ell_{0}, \ell_{1}, \ldots\right\}$ be a fixed alphabet of labels. Then, a labeled ordered tree on $\mathcal{L}($ tree, for short) is a labeled, rooted, connected directed acyclic graph $T=\left(V, E, B, L, v_{0}\right)$ with the following properties [3]. Each node $v \in V$ of $T$ is labeled by an element $L(v)$ of $\mathcal{L}$, and all node but the root $v_{0}$ have the unique parent by the child relation $E \subseteq V^{2}$. For each node $v$, its children are ordered from left to right by an indirect sibling relation $B \subseteq V^{2}$ [3]. Note that the term ordered means the order not on labels but on children.

The size of a tree $T$ is the number of its nodes $|T|=|V|$. Throughout this paper, we assume that a tree of size $k$ has the node set $V=\{1, \ldots, k\}$ and the nodes are ordered in the pre-order by the depth-first search order on $T$. We refer to the node $i$ as the $i$-th node of $T$. This assumption is crucial in our discussion. By this assumption, the root and the rightmost leaf of $T$, denoted by $\operatorname{root}(T)$ and $r m l(T)$, are always 1 and $k$, respectively. For a tree $T$ of size $k$, the rightmost branch of $T$, denoted by $R M B(T)$, is the path from the root 1 to the rightmost leaf $k$ of $T$.

We denote by $\mathcal{T}$ the class of all labeled ordered trees on $\mathcal{L}$. We also refer to $V, E, B$ and $L$ as $V_{T}, E_{T}, B_{T}$ and $L_{T}$, respectively, if it is clear from context.

Matching and Occurrences. Next, we define the notion of matching between two labeled ordered trees $T$ and $D$. A pattern tree $T$ matches a data tree $D$ if $T$ can be em-

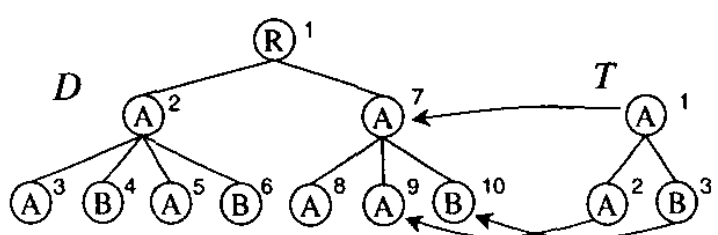

Figure 1. A data tree $D$ and a pattern tree $T$ on the set $\mathcal{L}=\{A, B\}$ of labels

bedded in $D$ with preserving the labels, the (direct) child relation, the (indirect) sibling relation by a non-collapsing mapping, that is, there exists some function $\varphi: V_{T} \rightarrow V_{D}$ that satisfies the following (i)-(iv) for any $v, v_{1}, v_{2} \in V_{T}$ :

(i) $\varphi$ is one-to-one.

(ii) $L_{T}(v)=L_{D}(\varphi(v))$.

(iii) $\left(v_{1}, v_{2}\right) \in E_{T}$ iff $\left(\varphi\left(v_{1}\right), \varphi\left(v_{2}\right)\right) \in E_{D}$.

(iv) $\left(v_{1}, v_{2}\right) \in B_{T}$ iff $\left(\varphi\left(v_{1}\right), \varphi\left(v_{2}\right)\right) \in B_{D}$.

Then, we say that $\varphi$ is a matching function of $T$ to $D$. or T occurs in D. We assume the emptr tree $\perp$ such that $|\perp|=$ 0 and $\perp$ matches to any tree at any node. An embedding of $T$ in $D$ w.r.t. $\varphi$ is the image $\varphi(T) \subseteq V_{D}$ of $T$ into $D$. whose induced subgraph is isomorphic to $T$. We define the root occurrence and the rightmost leaf occurrence of $T$ in $D$ w.r.t. $\varphi$ by the nodes $\varphi(1)$ and $\varphi(k)$ of $D$ to which the root and the rightmost leaf of $T$ map, respectively. If $\varphi$ is not irrelevant then we simply omit $\varphi$.

For example, Fig. 1 shows examples of labeled ordered trees $D$ and $T$ on $\mathcal{L}=\{A, B\}$. We see that the pattern tree $T$ matches the data tree $D$, where the matching is designated with a set of arrows from $T$ to $D$. The root occurrences of $T$ in $D$ are 2 and 7 , while the rightmost occurrences are 4,6 , and 10 .

Semi-structured Data Streams. Let $D$ be a labeled ordered tree, called a data tree with finite depth and possibly infinite width. Given a collection of trees as a data source, we always treat them as a single tree by combining trees with appending the imaginary common root. Recall that the nodes of $D$ are numbered in the preorder traversal of $D$.

We introduce a convenient sequential representation of labeled ordered trees. The depth of node $v$ of tree $T$ is the number of edges on the path from the root to $v$. The depthlabel representation of a node $v$ of $D$ is the pair $(d, \ell) \in$ $\mathbf{N} \times \mathcal{L}$ of the depth $d$ and the label $\ell$ of $v$. Then, a data tree $D$ is encoded as the sequence $\pi=\left(\left(d_{1}, \ell_{1}\right),\left(d_{2}, \ell_{2}\right), \ldots\right)$ of depth-label pairs corresponding to the nodes on the preorder traversal of $T$. This depth-label representation $\pi$ also linearly related to the open-close parentheses representation as in XML [19].

Conversely, we can uniquely decode a depth-label representation $\pi$ into a labeled ordered tree as follows. 
Definition $4([4,22])$ Let $S$ be a tree of size $k \geq 1$. Then, a rightmost expansion of $S$ is any tree $T$ of size $k+1$ obtained from $S$ by (i) attaching a new node $w$ with a label in $\mathcal{L}$ as a child of a parent node $p$ on the rightmost branch of $S$ so that (ii) $w$ is the rightmost sibling of $p$. Then, we say that $T$ is a successor of $S$, or $S$ is a predecessor of $T$. If the depth and the label of $w$ is $d \in \mathbf{N}$ and $\ell \in \mathcal{L}$, resp., then $T$ is called the $(d, \ell)$-expansion of $S$. The $(0, \ell)$-expansion of $\perp$ is the single node tree with label $\ell$.

Thus, the empty sequence $\varepsilon$ transforms to the empty tree $\perp$, and if the sequence $\pi$ transforms to a tree $S$, then the sequence $\pi \cdot(d, \ell)$ to the $(d, \ell)$-expansion of $S$. The notion of depth-label representation is motivated by the tree expansion technique $[4,22]$, and plays an important role in the following discussion.

For example, in the previous example of Fig. 1, the data tree $D$ transforms to the depth-label representation $\pi=$ $(0, R),(1, A),(2, A),(2, B),(2, A),(2, B),(1, A),(2, A)$, $(2, A),(2, B)$, and vice versa.

We model a semi-structured data stream as an infinite sequence of the nodes generated by the depth-first scanning of a possibly infinite data tree as follows. For a set $A$, we denote by $A^{\infty}$ the sets of all infinite sequences on $A$. A semi-structured data stream for $D$ is an infinite sequence $\mathcal{S}=\left(\boldsymbol{v}_{1}, \boldsymbol{v}_{2}, \ldots, \boldsymbol{v}_{i}, \ldots\right) \in(\mathbf{N} \times \mathcal{L})^{\infty}$, where for every $i \geq 1$, the $i$-th element $v_{i}=\left(d_{i}, \ell_{i}\right)$ is the depth-label representation of the $i$-th node $v_{i}=i$ of $D$. Then, $v_{i}$ is called the $i$-th node of $\mathcal{S}$ and $i$ is called the time stamp. The $i$-th left-half tree, denoted by $D_{i}$, is the labeled ordered tree that is the induced subgraph of $D$ consisting of the first $i$ nodes $\left(v_{1}, v_{2}, \ldots, v_{i}\right)$ of the traversal.

Online Data Mining Problems. Now, our online data mining problem is stated as follows. The definition of the frequency of a pattern $T$ at time $i$ will be specified later.

Definition 5 (Online Frequent Pattern Discovery from Semi-structured Data Streams) Let $0 \leq \sigma \leq 1$ be a nonnegative number called the minimum support. In our online mining protocol, for stages $i=1,2, \ldots$, an online mining algorithm $\mathcal{A}$ iterates the following process: $\mathcal{A}$ receives the $i$-th node $v_{i}$ from the stream $\mathcal{S}$, updates its internal state based on the first $i$ nodes $v_{1}, \ldots, v_{i}$ received so far, and then on request by a user $\mathcal{A}$ reports a set $\mathcal{F}_{i}$ of frequent patterns that appears in $D_{i}$ with frequency no less than $\sigma$.

The goal of an online algorithm is to continuously work on unbounded stream for arbitrary long time with bounded resources, and to quickly answer user's queries at any time.

We define the models of the frequency of patterns as follows. Let $i \geq 1$ be any time. For every $1 \leq j \leq i$, we define the indicator function $h i t_{j}^{(i)}(T)=1$ if the pattern $T$ has a root occurrence at the node $v_{j}$ in $D_{i}$. Otherwise, we define $h i t_{j}^{(i)}(T)=0$. For a technical reason, we require not only $\varphi(1)$ but also the whole $\varphi(T)$ to be contained in $D_{i}$.
Definition 6 Let $\mathcal{S}$ be a given semi-structured data stream and $T \in \mathcal{T}$ be any pattern. Below, count $_{i}(T)$ and freq $_{i}(T)$ denote the count and the frequency of $T$ at time $i$, resp.

- Online Model (OL). In this model motivated by Hidber [11], we count the number of distinct root occurrences of $T$ in $D_{i}$. The frequency of $T$ at time $i$ is:

$$
\text { freq }_{i}(T)=\frac{1}{i} \operatorname{count}_{i}(T)=\frac{1}{i} \sum_{j=1}^{i} h_{i} t_{j}^{(i)}(T)
$$

- Forgetting Model (FG). In the forgetting model, e.g., [21], the contribution of the past event decays exponentially fast. For positive number $0<\gamma<1$ called a forgetting factor, the frequency of $T$ is defined by:

$$
f r e q_{\gamma, i}^{\mathrm{fg}}(T)=\frac{1}{Z_{i}} \sum_{j=1}^{i} \gamma^{i-j} h i t_{j}^{(i)}(T) .
$$

Although we used a simplified normalization factor $Z_{i}=i$ instead of a more precise one $Z_{i}=$ $\sum_{j=1}^{i} \gamma^{i-j}$, most of the discussion in the later sections also holds.

A difference of above models is the speed of decay. Since the effect of a past event decays exponentially faster in FG than in OL, the former is more trend conscious than the latter. We can deal with the sliding window model in this framework in a similar manner. For details, see [5].

\section{Online Mining Algorithms}

In this section, we present an online algorithm StreamT for solving the online frequent pattern discovery problem from semi-structured data stream.

\subsection{Overview of the Algorithm}

In Fig. 2, we show our algorithm StreamT in the online model. Let $\mathcal{S}=\left(\boldsymbol{v}_{1}, \boldsymbol{v}_{2}, \ldots, \boldsymbol{v}_{i}, \ldots\right) \in(\mathrm{N} \times \mathcal{L})^{\infty}$ be a possibly infinite data stream for a data tree $D$. Through the stages $i=1,2, \ldots$, StreamT receives the $i$-th node $v_{i}=\left(d_{i}, \ell_{i}\right)$ from $\mathcal{S}$, updates a pool $\mathcal{C} \subseteq \mathcal{T}$ of candidate patterns and the internal state, and on request reports a set of frequent labeled ordered trees $\mathcal{F}_{i} \subseteq \mathcal{T}$ with frequency no less than a given threshold $0 \leq \sigma \leq 1$.

To continuously compute the set of frequent patterns on an unbounded stream, the algorithm uses a technique, similar to plane sweeping in computational geometry [8], to find all root occurrences of candidate patterns in $D$. A basic idea of our algorithm is explained as follows. To detect all embeddings of a set of patterns in $D$, we sweep a path from the root to the currently scanned node $v_{i}$, called the sweep branch, rightwards over the data tree $D$ by increasing the stage $i=1,2, \ldots$. While we sweep the plane, we keep track of all embedding of patterns that intersect the current sweep branch. 


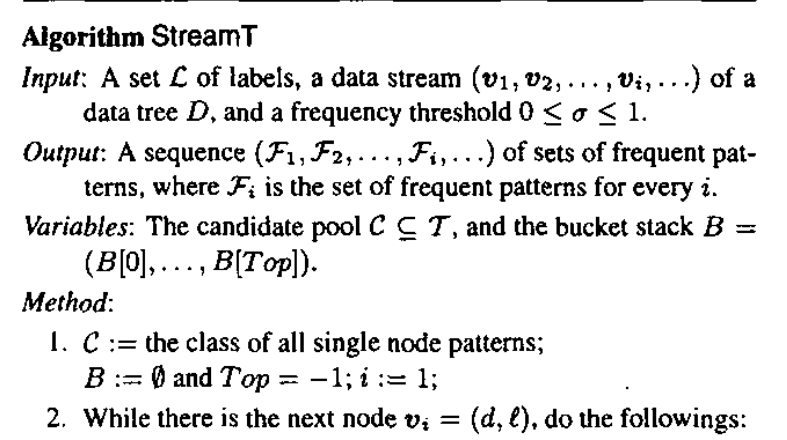

(a) Update the bucket stack $B[0] \cdots B[d-1]$ :

$$
(B, E X P):=\operatorname{UpdateB}(B, \mathcal{C},(d, \ell), i) ;
$$

(b) Update the candidate pool $\mathcal{C}$ and the bucket $B[d]$ :

$$
(B, \mathcal{C}):=\text { UpdateC }(E X P, B, \mathcal{C},(d, \ell), i) ;
$$

(c) Output the set $\mathcal{F}_{i}=\left\{T \in \mathcal{C} \mid\right.$ freq $\left._{i}(T) \geq \sigma\right\}$ of frequent patterns; $i=i+1$;

Figure 2. An online mining algorithm for semistructured data stream

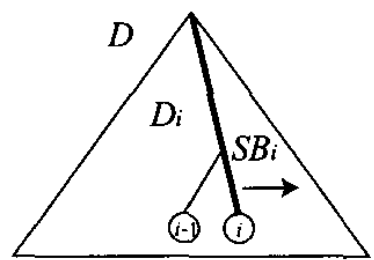

Figure 3. The $i$-th left-half tree $D_{i}$ and $i$-th sweep branch $S B_{i}$ for the data tree $D$

The algorithm incrementally maintains the following data structures during the computation.

- A set $\mathcal{C} \subseteq \mathcal{T}$ of patterns, called the candidate pool.

- A stack $B=(B[0], B[1], \ldots, B[T o p])$ of buckets, called the sweep branch stack (SB-stack, for short).

For each candidate $T \in \mathcal{C}$, the following features are associated: A counter count $(T)$ of the root occurrences of $T$ in $D_{i}$. A vector Rto $_{T}=\left(\right.$ Rto $_{T}[0]$, Rto $\left.T[1], \ldots\right)$ of the latest root occurrences Rto $_{T}[d]=\rho$ of $T$ with depth $d$.

\subsection{Incremental Pattern Discovery Using Tree Sweeping}

To keep track of all embeddings of candidate pattems, we do not need the whole information on them. Instead, we record the information on the intersections of these embedding and the current sweep branch at every stage.

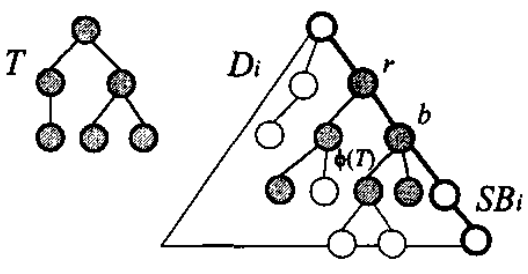

Figure 4. The root and the bottom occurrences $r$ and $b$ of pattern $T$ on $D_{i}$ w.r.t the sweep branch $S B_{i}$ with matching $\phi$.

Let $i \geq 1$ be any stage. In what follows, we denote by $v_{i}, D_{i}$ and $S B_{i}$ the present node, the left-half tree and the sweep branch at stage $i$. In other words, $S B_{i}$ is the rightmost branch of $D_{i}$.

For pattern $T$, let $\varphi(T)$ be an embedding of $T$ with some matching $\varphi: V_{T} \rightarrow V_{D}$ of $T$ to $D_{i}$. Since an embedding of a tree is also an ordered tree in $D_{i}$, we can define the rightmost branch, denoted by $R M B(\varphi(T)) \subseteq V_{D}$, of $\varphi(T)$ in $D_{i}$. During the scan of $D$, the sweep branch $S B_{i} \subseteq V_{D}$ may have nonempty intersection $S B_{i} \cap R M B(\varphi(T))$ with $R M B(\varphi(T))$.

Lemma 1 For any embedding $\varphi(T)$ of a pattern $T$ and the sweep branch $S B_{i}$, the intersection $S B_{i} \cap R M B(\varphi(T))$ is a consecutive path in $D$.

From the above lemma, we define the root and the bottom occurrences of $T$ w.r.t. $\varphi$ to be the highest and the lowest nodes in the intersection $S B_{i} \cap R M B(\varphi(T))$ (Fig. 4). We can easily see that if the intersection $S B_{i} \cap$ $R M B(\varphi(T))$ is contained in $S B_{i}$ then the corresponding bottom occurrence becomes the rightmost occurrence of $T$ w.r.t. $\varphi$. The next lemma gives an incremental characterization of the rightmost occurrences, which enables us to detect all rightmost occurrences of candidate patterns by maintaining all bottom occurrences of their predecessors on the sweep branch using the SB-stack.

Lemma 2 Let $T \in \mathcal{T}$ be any pattern of size $k>1$. At every time $i \geq 1, T$ has a rightmost occurrence at the current node $v_{i}$ in $D_{i}$ iff there exists some pattern $S$ of size $(k-1)$ that has a bottom occurrence at the parent of $v_{i}$ in $D_{i}$ and such that $T$ is the $(d, \ell)$-expansion of $S$, where $d$ is the depth of the rightmost leaf $k$ of $T$ from its root and $\ell=L\left(v_{i}\right)$ is the label of $v_{i}$. This is also true even if $k=1$.

To implement this idea, we use the sweep branch stack $B=(B[0], B[1], \ldots, B[T o p])$ to record the intersections of embeddings of patterns with the current sweep branch $S B_{i}$. Top $\geq 0$ is the length of $S B_{i}$. Each bucket $B[b](0 \leq$ $b \leq T o p)$ contains a set of triples of the form $\tau=(T, r, b)$ such that pattern $T$ has the root and the bottom occurrences 
of the depths $r$ and $b$, respectively, on $S B_{i}$. For each bucket $B[d]$, the time stamp $B[d]$.time $\in \mathbf{N}$ of the last time is associated with the bucket.

For any stage $i \geq 1$, the SB-stack $B=$ $(B[0], B[1], \ldots, B[T o p])$ is said to be up-to-date w.rt. the present sweep branch $S B_{i}$ if $T o p$ is the length of $S B_{i}$, and for every $0 \leq b \leq T o p$, the bucket $B[b]$ contains all triples $(T, r, b) \in \mathcal{T} \times \mathbf{N} \times \mathbf{N}$ for some $T \in \mathcal{C}$ and $r \in \mathbf{N}$ such that the pattem $T$ appears in $D_{i}$ and has the root and the bottom occurrences on $S B_{i}$ of the depths $r$ and $b$, respectively (Fig. 4). Then, we also say that each bucket $B[b]$ is up-to-date if no confusion arises. Note that the up-to-date stack is unique at time $i$. Now, we give a characterization of the contents of the current SB-stack $B_{i}$ inductively.

Lemma 3 Let $i \geq 1$ and $v_{i}=(d, \ell)$ be the current node of the data stream $\mathcal{S}$ for $D$. Let $B_{k}=$ $\left(B_{k}[0], B_{k}[1], \ldots, B_{k}\left[T o p_{k}\right]\right)$ be the $S B$-stack at time $k \in$ $\{i-1, i\}$. Suppose that both of the $S B$-stacks $B_{i}$ and $B_{i-1}$ are up-to-date. Then, the following $1-4$ hold:

1. For any $0 \leq b<d-1, \tau \in B_{i}[b]$ if and only if $\tau \in B_{i-1}[b]$.

2. For $b=d-1, \tau \in B_{i}[d-1]$ if and only if either (i) or (ii) below holds:

(i) $\tau \in B_{i-1}[d-1]$.

(ii) $\tau$ is represented as $\tau=(T, r, d-1)$ for some tuple $(T, r, b) \in B_{i-1}[d] \cup \cdots \cup B_{i-1}\left[T o p_{i-1}\right]$ such that $r \leq d \leq b$.

3. $\tau \in B_{i}[d]$ if and only if $\tau=(T, r, b)$ and either (i) or (ii) below holds:

(i) $T$ is the single node tree with the label $\ell$.

(ii) $T$ is the $(d-r, \ell)$-expansion of $S$ for some triple $(S, r, d-1) \in B_{i}[d-1]$.

4. For any $b>d, B_{i}[b]$ is undefined.

Proof. Case 1, 2, 3(i) and 4 are obvious. For case 3(ii), suppose that $E_{T}$ is an embedding of $T$ in $D$ and its right-branch embedding intersects $S B_{i}$ with the bottom depth $b=d$. Then, $T$ has the rightmost occurrence at the current node $v_{i}=i$. Let $C_{T}$ is the tree obtained from $E_{T}$ by removing $v_{i}$. Then, $C_{T}$ is an embedding of the predecessor of $T$ with the rightmost occurrence at the parent, say $w$, of $v_{i}=i$. Since the depth of the parent $w$ is $d-1$, the corresponding triple $(S, r, d-1)$ for $C_{T}$ belongs to $B_{i}[d-1]$ where $T$ is the $(d-r, \ell)$-expansion of $S$.

Fig. 5 illustrates how to update the sweep branch stack $B_{i-1}$ based on Lemma 3. Suppose that we receive the $i$-th node $(d, \ell)$ from a data stream. Then, the triples in UNCHANGE buckets, i.e., $B[0] \cup \cdots \cup B[d-1]$, stay unchanged. The buckets in $B[d] \cup \cdots \cup B[T o p]$ are partitioned

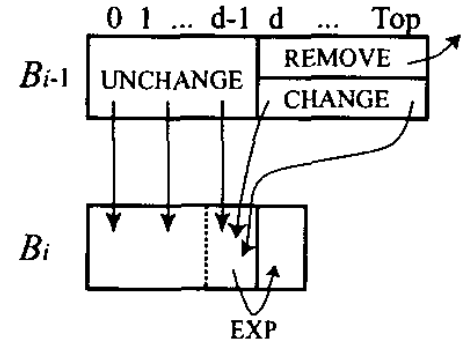

Figure 5. SB-stacks from time $i-1$ to $i$

Algorithm UpdateB $(B, \mathcal{C},(d, \ell), i)$

Input: A bucket stack $B=(B[0], B[1], \ldots, B[$ Top $])$, a candidate pool $\mathcal{C}$, a depth-label representation $v_{i}=(d, \ell) \in \mathbf{N} \times \mathcal{L}$ of the $i$ th node of the data stream $\mathcal{S}$, and the current time $i$;

Output: A set $E X P \subseteq \mathcal{T} \times \mathbf{N} \times \mathbf{N}$ of triples ;

Method:

1. If $d \leq T_{O p}$, then do the followings:

$-B E L O W:=B[d] \cup \ldots \cup B[T o p]$;

- ${ }^{*}$ Discard the triples below the branching point */ REMOVE $:=\{(T, r, b) \in B E L O W \mid r \geq d\} ;$

$-I^{*}$ Collect the triples across the branching point $* /$ CHANGE $:=\{(T, r, b) \in B E L O W \mid r \leq d-1\} ;$

$-{ }^{*}$ Change the bottom occurrences of the triples */ $B[d-1]:=B[d-1\} \cup\{(T, r, d-1) \mid(T, r, b) \in$ CHANGE \};

2. EXP:=\{(T, $\left.\left.T_{\ell}, d\right)\right\}$, where $T_{\ell}$ is the single node tree with the label $\ell$;

3. For each $(S, r, d-1) \in B[d-1]$ do the followings:

$-T$ is the $(d-r, \ell)$-expansion of $S$;

$-E X P:=E X P \cup\{(T, r, d)\}$;

4. Return $(B, E X P)$;

\section{Figure 6. Updating the SB-stack}

into REMOVE and CHANGE. The triples in REMOVE buckets are discarded, and triples in CHANGE buckets move to the bucket $B[d-1]$. For all triples in $B[d-1]$, we apply the rightmost expansion and then insert obtained expansions into EXP.

In Fig. 6, we show the algorithm UpdateB for incrementally updating the SB-stack. At any stage $i \geq 1$, UpdateB updates the first $d-1$ buckets $B_{i}[0] \cdots B_{i}[d-1]$ of new one. The $d$-th bucket is not immediately updated, but the updated contents are separately returned as $E X P$ for computing the bucket $B_{i}[d]$ in further processing. The following corollary is immediately from Lemma 3.

Corollary 4 For every time invoked in the while loop in StreamT of Fig. 2 at time $i \geq 1$ with the current node $\boldsymbol{v}_{i}=(d, l)$, the algorithm $\operatorname{Update} \mathrm{B}(B, \mathcal{C},(d, \ell), i)$ returns 
the followings: (i) The sequence $B[0], \ldots, B[d-1]$ of buckets that are up-to-date at time $i$ up to depth $d-1$. (ii) The set EXP of all triples corresponding to the bottom occurrences on $S B_{i}$ whose depth is $d$ and predecessors belong to $\mathcal{C}$.

\subsection{Duplicate Detection for the Root Occurrences}

From the observations above, the algorithm UpdateB (Fig. 6) detects all rightmost leaf occurrences of the patterns whose predecessors belong to $\mathcal{C}$ at Step 2 and Step 3 .

Then, the next step is to compute the corresponding root occurrences from these rightmost occurrences. Let $b \in V_{D}$ be a rightmost occurrence of pattern $T$ whose triple $(T, r, b)$ is detected at Step 2 or Step 3 of UpdateB. Recall that a list Rto $T=\left(\right.$ Rto $_{T}[0]$, Rto $\left._{T}[1], \ldots\right)$ is associated with each $T \in \mathcal{C}$ and it is initially empty. Then, we can obtain the root occurrence corresponding to the triple by the following procedure FindRootOcc:

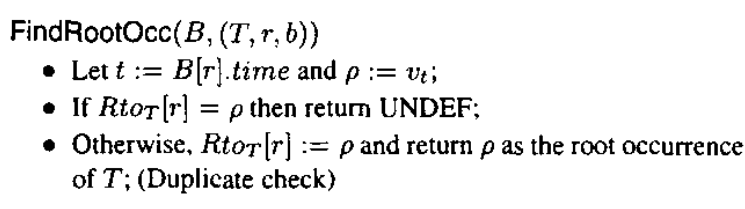

\section{Figure 7. Finding root occurrences}

It is easy to observe that FindRootOcc correctly finds the root occurrence as follows. If the sweep branch $S B_{i}$ intersects an embedding of $T$ w.r.t. a matching $\varphi$ then it also intersects the root occurrence of $T$ w.r.t. $\varphi$, and thus the component $r$ of $(T, r, b)$ correctly gives the depth of a root occurrence, say, $w \in V_{D}$. By definition, $B[r]$.time stores the time stamp, say $t$, of the node on $S B_{i}$ whose depth is $r$ when it is first encountered. Thus, $v_{t}=w$ gives the root occurrence corresponding to the triple.

Furthermore for a fixed $r$, any node $w^{\prime}$ occupies the bucket $B[r]$ in a consecutive period during the scanning of $\mathcal{S}$. Thus, it is sufficient to record the last root occurrence of depth $r$ for each depth $r \geq 0$ in order to check the duplication of the occurrences. Hence, we see that FindRootOcc correctly detect the root occurrence of candidate patterns without duplicates.

\subsection{Candidate Management}

The algorithm StreamT stores candidate patterns in a candidate pool $\mathcal{C} \subseteq \mathcal{T}$. Fig. 8 shows an algorithm UpdateC for managing $\mathcal{C}$ by updating the frequency count of each patterns. A root occurrence has monotonicity that if pattern $T$ is a rightmost most expansion of pattern $S$ then the root count of $S$ is greater than or equal to the root count of $T$. Based on this observation, the algorithm UpdateC uses a
Algorithm UpdateC $(E X P, B, \mathcal{C},(d, \ell), i)$

Input: A set $E X P$ of triples, a bucket stack $B=$ $(B[0], B[1], \ldots, B[T o p])$, a candidate pool $\mathcal{C}$, the $i$-th node $\boldsymbol{v}_{i}=(d, \ell) \in \mathbf{N} \times \mathcal{C}$ of the data stream, and the time $i$;

Output: The updated pair $(B, \mathcal{C})$;

Method:

1. /* Increment candidates */

For each triple $(T, r, b) \in E X P$, do:

If $\rho:=$ FindRootOcc $(\mathrm{B},(\mathrm{T}, \mathrm{r}, \mathrm{b}))$ is not UNDEF then

- If $T \in \mathcal{C}$ then $\operatorname{count}(T):=\operatorname{count}(T)+1$;

- If $T \notin \mathcal{C}$ and the predecessor of $T$ is frequent, then $\operatorname{count}(T):=1$ and $\mathcal{C}:=\mathcal{C} \cup\{T\}$;

2. $B[d]:=\emptyset ;$ Top $:=d ; B[d]$.time $:=i$;

$\operatorname{freq}(T):=\operatorname{count}(T) / i$

3. /*Delete candidates */

For each pattern $T \in \mathcal{C}$ and the predecessor of $T$ is infrequent at time $i$ and frequent at time $i-1$,

$-\mathcal{C}=\mathcal{C} \backslash\{T\}$

4. $/ *$ Insert candidates in $B[d] * /$

For each triple $(T, r, b) \in E X P$

- If $T \in \mathcal{C}$ then $B[d]:=B[d] \cup\{(T, r, b)\}$;

5. Return $(B, \mathcal{C})$;

\section{Figure 8. Updating the candidate pool}

candidate management policy similar to Hidber [11], which is summarized as follows.

- Initialize. We insert all single node patterns into $\mathcal{C}$. This is done at Step 1 of the algorithm StreamT

- Increment. We increment $\operatorname{count}(T)$ for all pattern trees $T \in \mathcal{C}$ that has the rightmost occurrence at the current node $v_{i}$, i.e., $\operatorname{count}(T)=\operatorname{count}(T)+1$.

- Insert. We insert a pattern of size more than one if its unique predecessor $S$ is already contained in $\mathcal{C}$ and becomes frequent, i.e., freq $(S) \geq \sigma$ based on the monotonicity of freq $(S)$ w.r.t. rightmost expansion. This is an on-demand version of the insertion policy of [11]. If some pattern becomes frequent then insert all of its successors to the candidate pool.

- Delete. We delete a pattern $T$ from $\mathcal{C}$ when its unique predecessor $P$ becomes infrequent, i.e., freq $(T)<\sigma$. To be consistent to the initialization and the insertion policy, we do not delete any single nodes. As suggested in [11], we postpone the deletion of the patterns from $\mathcal{C}$ until the algorithm requires additional space.

As summary, our algorithm StreamT tries to maintain the negative border [17], the set of all patterns that are infrequent but whose predecessors are frequent. 


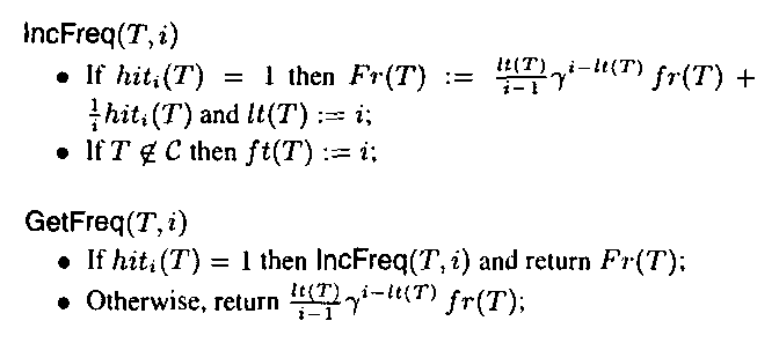

Figure 9. Updating and Computing the Frequency

\subsection{Time Analysis}

We give theoretical analysis of the update time of the algorithm StreamT at stage $i$. Let $B_{i-1}$ be the previous SB-stack of top Top and $N_{j}=\left|B_{i-1}[j]\right|$ be the number of triples in the $j$-th bucket. If a node of depth $d$ is received, then UpdateB updates the SB-stack in $O\left(\sum_{j=d-1}^{T o p} N_{j}\right)$ time. Then, UpdateC updates pattern pool $\mathcal{C}$ in $O(k C+D)$ time, where $k$ is the maximum pattern size, $C$ is the number of candidates in $\mathcal{C}$ that occur at the current node $v_{i}$ by the rightmost leaf occurrence, and $D$ is the number of removed candidates in the stage.

\section{Modification to the Forgetting Model}

The algorithm StreamT in Fig. 2 is an online algorithm for the online frequent pattem discovery problem in the online model of Definition 6. Now we present modification of StreamT to the forgetting model also introduced in Definition 6.

Recall that in the forgetting model, the contribution of the past event decays exponentially fast. For a forgetting factor $0<\gamma<1$, the frequency of $T$ at time $i$ is given by Eq. 1 in Section 2. At first look, implementation of the forgetting model is as easy as the online model above because they only differ in the definition of the frequency. In the forgetting model, however, the frequency at time $i$ depends on all of the weights $\gamma^{i-j}$ of past events changing as $i \geq 1$ goes larger. Thus, it seems that an algorithm have to maintain all of these weights at every time. Fortunately, this is not true.

We abbreviate the frequency freq $_{\gamma, i}^{\mathrm{fg}}(T)$ and the event $h i t_{j}^{(i)}(T)$, respectively, to $f r_{i}$ and $h i t_{j}$. Below, we give an incremental method to compute the frequency. Let $l t(i)=$ $\max \left\{j \leq i \mid\right.$ hit $\left._{j}=1\right\}$ be the last time stamp at which $T$ appeared. Then, we have the following lemma.

Lemma 5 For every $T \in T$ and every $i \geq 1$, we have the recurrence

$$
\begin{aligned}
& f r_{0}=0 \\
& f r_{i}=\frac{l t(i)}{i-1} \gamma^{i-l t(i)} f r_{l t(i)}+\frac{1}{i} h i t_{i} \quad(i>0)
\end{aligned}
$$

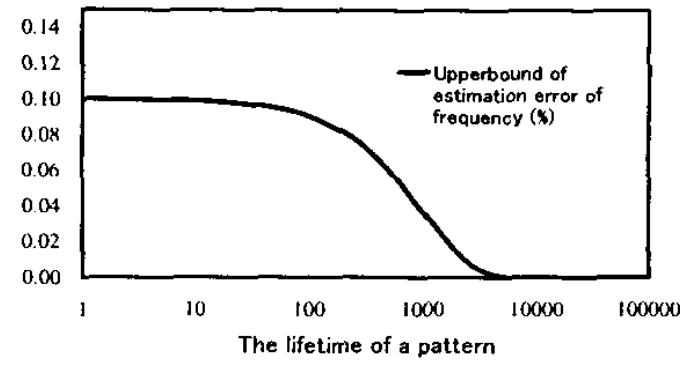

Figure 10. The upper bound of the frequency error against the life time with $\gamma=0.999$

Proof. We first derive the recurrence for the consecutive steps. Then, derive the recurrence of the lemma by expanding $f r_{i}$ using $l t(i)$. Since $h i t_{u}=0$ for any $u$ with $l t(i)<u<i$, the claim immediately follows.

Now, we present a modified version of StreamT in the forgetting model. We modify the algorithms StreamT and UpdateC (Fig. 8) as follows. StreamT maintains the three parameters $f r(T), f t(T), l t(T)$, the frequency, the first and the last time stamps of the updates for $T$. UpdateC uses IncFreq to update these parameters whenever a root occurrence of pattern $T$ is detected at Step 1, and Stream uses GetFreq to compute the frequency of $T$ whenever it receives a request at Step 2(c). We can see IncFreq $(T, i)$ and GetFreq $(T, i)$ can be executed in constant time when invoked by these procedures.

Then, we will present an upper bound of the error to estimate the true frequency of a pattern in this model. The life time of a pattem $T$ is the period $\Delta i=i-f t(T) \geq 0$. The following theorem says that the error becomes exponentially smaller in the forgetting model as the life time $\Delta i$ of $T$ goes longer.

Theorem 6 Let $i \geq 1,0<\gamma<1$, and $\varepsilon=1-\gamma$. For any $T \in \mathcal{C}$ with the life time $\Delta i=i-f t(T)$ at time $i$, the following inequality holds:

$\operatorname{GetFreq}(T, i) \leq \operatorname{freq}_{\gamma, i}^{\mathrm{fg}}(T) \leq \operatorname{GetFreq}(T, i)+\frac{1}{\varepsilon i} e^{-\varepsilon \Delta i}$.

Proof. By standard arguments using elementary calculus, Eqs. $1-\gamma^{x} \leq 1$ and $(1-\varepsilon)^{x} \leq e^{-\varepsilon x}(x \geq 0)$.

In Fig. 10, we plot the upper bound of the frequency error given by Theorem 6 varying the life time from $\Delta i=1$ to $\Delta i=100,000$, where $\gamma=0.999$ and $i=1000,000$. A half-value period is about seven thousands for $\gamma$.

\section{Experimental Results}

In this section, we present preliminary experimental results on real-life datasets to evaluate the algorithm 


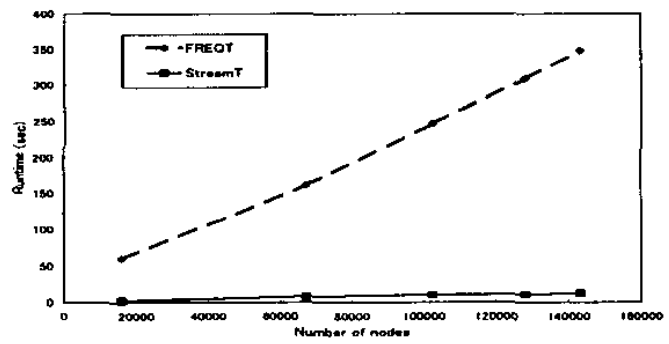

Figure 11. the online scale-up experiment

StreamT. We prepared a dataset Citeseers by collecting cgi-generated Web pages from an online bibliographic archive Citeseers '. This dataset consists of 189 HTML documents of 5.6MB and its data tree had 143,250 nodes. All experiments were performed on PC (PentiumIII 500MHz, 512MB RAM, Windows2000) and the algorithm was implemented in Java (SUN JDKI.3.1, JIT compiler).

We studied the scalability of StreamT. Fig. 11 shows the running times of the online version StreamT and the offline version FREQT [4] of frequent tree miners with the same frequency threshold $\sigma=2(\%)$ on the data stream for the dataset Citeseers varying the data size from $16 \mathrm{~K}$ (nodes) to $143 \mathrm{~K}$ (nodes).

From this experiment, the proposed online algorithm StreamT seems to be much more efficient than the offline algorithm FREQT. However, this is possibly because our algorithm computes approximate answers due to candidate management policy in Sec. 3.4 due to [11] and two algorithms may generate different sets of patterns. Therefore, to compare the performance of those algorithms, we require further research.

\section{Conclusion}

In this paper, we studied an online data mining problem from unbounded semi-structured data stream. We presented efficient online algorithms that are continuously working on an unbounded stream of semi-structured data with bounded resources, and find a set of frequent ordered tree patterns from the stream on request at any time.

Our labeled ordered trees can be seen as a generalization of serial episodes of Mannila et al. [14], and of itemsets and sequential pattems with a pre-processing of data as used for encoding XML-attributes in [4]. Thus, it will be an interesting problem to study the relationship of our algorithms to other online algorithms for classes of patterns such as sequential patterns and episodes.

It is also a future problem to examine the online.property of the proposed algorithms using long and trend-changing semi-structured data streams in the real world.

\footnotetext{
${ }^{1}$ http://citeseer.nj.nec.com/
}

\section{Acknowledgments}

Hiroki Arimura is grateful to Masaru Kitsuregawa for his direction of the author's interests to online data processing. The authors would like to thank Hiroshi Motoda, Takeshi Tokuyama, Akihiro Yamamoto, Yoshiharu Ishikawa, and Mohammed Zaki for fruitful discussions on web mining.

\section{References}

[1] K. Abe, S. Kawasoe, T. Asai, H. Arimura, and S. Arikawa. Optimized Substructure Discovery for Semi-structured Data, In Proc. PKDD'02, 1-14, LNAI 2431, Springer, 2002.

[2] S. Abiteboul, P. Buneman, D. Suciu, Data on the Web, Morgan Kaufmann, 2000.

[3] Aho, A. V., Hopcroft, J. E., Ullman, J. D., Data Structures and Algorithm,s, Addison-Wesley, 1983.

[4] T. Asai, K. Abe, S. Kawasoe, H. Arimura, H. Sakamoto, and S. Arikawa. Efficient Substructure Discovery from Large Semistructured Data. In Proc. the 2nd SIAM Int'l Conf. on Data Mining (SDM2002), 158-174, 2002.

[5] T. Asai, K. Abe, S. Kawasoe, H. Arimura, H. Sakamoto, and S. Arikawa. Online Algorithms for Mining Semi-structured Data Stream, DOI Technical Report, Dept. of Informatics, Kyushu Univ., DOI-TR 21l, June 2002. ftp://ftp.i.kyushuu.ac.jp/pub/tr/trcs211.ps.gz

[6] R. J. Bayardo Jr., Efficiently Mining Long Patterns from Databases, In Proc. S/GMOD 98, 85-93, 1998.

[7] G. Cong, L. Yi, B. Liu, K. Wang, Discovering Frequent Substructures from Hierarchical Semi-structured Data, In Proc. SDM2002, $175-192,2002$

[8] M. de Berg, M. van Kreveld, M. Overmars, O. Schwarzkopf, Computational Geometry, Algorithms and Applications, Springer, 2000.

[9] L. Dehaspe, H. Toivonen, R. D. King, Finding Frequent Substructures in Chemical Compounds, In Proc. KDD-98, 30-36, 1998.

[10] P. B. Gibbons and Y. Matias, Synopsis Data Structures for Massive Data Sets. In External Memory Algorithms, DIMACS Series in Discr. Math. and Theor. Compt. Sci., Vo!. S0, AMS, 39-70, 2000.

[11] C. Hidber, Online Association Rule Mining, In Proc. SIGMOD'99 145-156, 1999

[12] P. Kilpelainen, H. Mannila, Ordered and Unordered Tree Inclusion, SIAM J. Comput, 24(2), 340-356, 1995.

[13] M. Kuramochi, G. Karypis, Frequent Subgraph Discovery, In Proc. ICDM Ol, 313-320, 2001

[14] H. Mannila, H. Toivonen, and A. I. Verkamo, Discovering Frequent Episode in Sequences, in Proc. KDD-95, 210-215, AAAl, 1995.

[15] T. Matsuda, T. Horiuchi, H. Motoda, T. Washio, K. Kumazawa, N. Arai, Graph-Based Induction for General Graph Structured Data, In Proc. DS'99, 340-342, 1999.

[16] T. Miyahara, Y. Suzuki, T. Shoudai, T. Uchida, K. Takahashi, H. Ueda, Discovery of Frequent Tag Tree Patterns in Semistructured Web Documents. In Proc. PAKDD-2002, 341-355, 2002.

[17] S. Parthasarathy, M. J. Zaki, M. Ogihara, S. Dwarkadas, Incremental and Interactive Sequence Mining, In CIKM'99, 251-258, 1999.

[18] R. Rastogi, Single-Path Algorithms for Querying and Mining Data Streams, In Proc. SDM2002 Workshop HDM'02, 43-48, 2002.

[19] W3C, Extensive Markup Language (XML) 1.0 (Second Edition), W3C Recommendation, 06 October 2000. http: //www . w3 . org/TR/REC-xml

[20] K. Wang, H. Liu, Discovering Structural Association of Semistructured Data, TKDE, 12(2), 353-371, 2000.

[21] K. Yamanishi, J. Takeuchi, A Unifying Framework for Detecting Outliers and Change Points from Non-Stationary Time Series Data, In Proc. SIGKDD-2002, ACM, 2002.

[22] M. J. Zaki. Efficiently mining frequent trees in a forest, In Proc, SIGKDD-2002, ACM, 2002 